\title{
The effect of COVID-19 derived cytokine storm on cancer cells progression: double-edged sword
}

\author{
Mohammad Heydarian ${ }^{1} \cdot$ Mohammadjavad Mohammadtaghizadeh $^{2} \cdot$ Mahboobeh Shojaei $^{3}$. \\ Marziyeh Babazadeh ${ }^{4}$. Sadegh Abbasian ${ }^{5}$. Mehran Amrovani ${ }^{3}$ (D)
}

Received: 17 July 2021 / Accepted: 1 October 2021 / Published online: 16 October 2021

(c) The Author(s), under exclusive licence to Springer Nature B.V. 2021

\begin{abstract}
Objective Severe acute respiratory syndrome coronavirus 2 (SARS-COV2) was first detected in Wuhan, China in December, 2019. The emerging virus causes a respiratory illness, that can trigger a cytokine storm in the body.

Method Cytokine storm in patient's body is associated with severe forms of disease. It is one of the main complications of coronavirus disease-2019 (COVID-19), in which immune cells play a major role. Studies have shown immune cells in the tumor environment can be effective to induce resistance to chemotherapy in cancer patients.

Result Therefore, considering the role of immune cells to induce cytokine storm in COVID-19 patients, and their role to cause resistance to chemotherapy, they are effective on disease progression and creation of severe form of disease.

Conclusion By examining the signaling pathways and inducing resistance to chemotherapy in tumor cells and the cells affect them, it is possible to prevent the occurrence of severe forms of the disease in cancer patients with COVID-19; it is applicable using target therapy and other subsequent treatment strategies.
\end{abstract}

Keywords Cytokine storm · Chemotherapy resistance $\cdot$ Pathogenesis $\cdot$ COVID-19

Mehran Amrovani

e.amrovani@gmail.com

Mohammad Heydarian

reza.heidarian.1382@gmail.com

Mohammadjavad Mohammadtaghizadeh

dr.taghizadeh87@gmail.com

Mahboobeh Shojaei

mahboobehshojaei22@yahoo.com

Marziyeh Babazadeh

marziyehbabazadehbio@gmail.com

Sadegh Abbasian

abbasian_sadegh@yahoo.com

Lorestan University of Medical Sciences, Khorramabad, Iran

2 Atherosclerosis Research Center, Ahvaz Jundishapur University of Medical Sciences, Ahvaz, Iran

3 High Institute for Education and Research in Transfusion Medicine, Tehran, Iran

4 Department of Biochemistry, School of Medicine, Shiraz University of Medical Sciences, Shiraz, Iran

5 Department of Laboratory Science, School of Paramedical Sciences, Ilam University of Medical Sciences, Ilam, Iran

\section{Introduction}

In late December 2019, an outbreak of pneumonia associated with a novel coronavirus, coronavirus disease-2019 (COVID-19), was first reported in Wuhan, Hubei Province, China; it quickly spread in all the countries around the world. The virus targets human respiratory system and has a relatively high mortality rate. Fever, cough, musculoskeletal and gastrointestinal symptoms and fatigue are the common symptoms of virus infection [1,2].

Accumulating evidence indicates cytokine storm syndrome may be present in the severely ill COVID-19 patients; it is a sign of immune dysregulation [3]. It has been shown to be associated with poor outcomes in COVID-19 patients. Cytokine storm is characterized by secretion of interleukins, interferons, chemokines, tumor-necrosis factors (TNF) and several other inflammatory mediators [4]. Plasma concentration of multiple inflammatory cytokines, such as granulocyte-macrophage colony-stimulating factor (GM-CSF), interleukin-6 (IL-6), tumor necrosis factor-alpha (TNFa), IL-2, 7, 10, and granulocyte colony-stimulating factor (G-CSF) increases following Severe acute respiratory syndrome coronavirus 2 (SARS-CoV-2) infection. 
In addition, it is demonstrated that peripheral inflammatory monocytes and pathogenic $\mathrm{T}$ cells might induce cytokine storms in severe COVID-19 patients [5, 6]. Various conditions including malignancies, sepsis, macrophage activation syndrome (MAS), systemic juvenile idiopathic arthritis (SJIA), cytokine release syndrome (CRS) and acute respiratory distress syndrome (ARDS) are generated by cytokine storm [7].

A higher risk of severe stages of COVID-19 infection is reported in actively treated cancer patients. It is also shown, that unlike the events in severely ill COVID-19 patients, cancer development is usually associated with immune responses dampen; it is portrayed via the increased secretion of anti-inflammatory cytokines, repressed induction of proinflammatory mediators and enhanced population of immunosuppressive leukocytes [8]. According to these reports, the cytokine storm threatens life in COVID-19 patients, but it can be a therapeutic option in cancer patients. In this study, the pathophysiology of cytokine storm has been studied in response to COVID-19 infection in malignancies.

\section{Cytokine release: protective versus pathogenesis}

Cytokine storm occurs with a sudden increase in the level of pro-inflammatory cytokines such as IL-1, IL-6, and TNF-a. These cytokines are among the most important pro-inflammatory ones; they considered as the innate immunity, and are produced by cells such as macrophages and inflamed endothelial cells. Increased level of these cytokines in blood leads to the summoning of immune cells to the inflamed and infected sites, which in turn leads to tissue damage, organ failure and eventually death $[9,10]$.

Some cytokines have the protective role, while others have the pathological role. Cytokine storm has also been reported in COVID-19 patients; serological assessments of plasma indicates increased levels of IL-1b, IL-6, IL-10 and TNF- $a$ in the cited patients. Studies have also shown, that increase in these cytokines leads to disease progression and worsening patients' clinical conditions, which ultimately leads to survival reduction $[11,12]$. Despite various studies, the molecular mechanisms of these cytokines (IL-1, IL-6 and TNF-a) in relation to the pathogenesis of malignancy in COVID-19 patients have not been fully identified yet.

\section{IL-6}

IL-6 is a pleiotropic cytokine, that has a wide range of biological activities, including: immune regulation, hematopoiesis, inflammation, effects on metabolic, proliferative and regenerative processes. It is produced by various cells in the tumor microenvironment (TME), and plays an important role in the expansion and differentiation of tumor cells [13].

IL-6 binds to the IL-6R (IL-6 Receptor) on the surface of target cell to form a heterohexameric complex, consisting of two molecules IL-6 and IL-6R, together with the IL-6 receptor subunit (gp130). In the downstream, the signaling pathway of this cytokine is activated by the JAK/STAT pathway. This pathway is also involved in a process called Epithelial Mesenchymal Transition (EMT), by activating a series of transcription factors.

Snail, Zeb1, JUNB and Twist1 are among the most important copying factors in the EMT induction pathway (EMT-TF) [14]. Snail transcription factor via Smad1/AKT/ GSK3 $\beta$ signaling pathway and increased expression of Nanog molecule can induce EMT in cell. Regarding other effective factors in EMT, the Zeb1 factor also applies a positive feedback to HAS2 molecule; also, JUNB factor can lead to EMT process by activating the TGF- $\beta$ molecule, and using its related signaling pathway [15-17].

Finally, Twist 1 factor can induce EMT process in cells by reducing the $\mathrm{H} 2 \mathrm{AX}$ expression, and subsequently by increasing the expression of VCAN, THBS1, TGFB2, ITGB4, SERPINE genes, and reducing the expression of CDH1, CTNNB1, OCLN genes [18]. EMT status plays an important role in resistance to chemotherapy, so the JAK/ STAT signaling pathway through this condition can lead to resistance to chemotherapy in patients with malignancy.

On the other hand, the heterohexameric complex IL-6/ IL-6R/gp130 can induce PI3K/AKT and MAPK/ERK signaling pathways in its downstream. These pathways also lead to the EMT status by applying Snail and Slug transcription factors, and increasing the expression of E-Cadherin and $\mathrm{N}$-cadherin molecules; by inducing this process in cells, they lead to resistance to chemotherapy in people with malignancy [19]. Studies show an increase in IL-6 levels in cancer patients with COVID-19, indicating a cytokine storm [20, 21]. It is hypothesized that, due to cytokine storms, elevated IL-6 levels in COVID-19 cancer patients and using the mentioned pathways in them, the phenomenon of resistance to chemotherapy drug occurs; it is accompanied by therapeutic effect decrement, increased mortality and survival reduction in patients.

\section{IL-1}

The IL- 1 family includes IL-1a, IL-1 $\beta$, and IL-1 Receptor Antagonist (IL1-Ra). IL-1 $\alpha / \beta$ binds to the IL- 1 type one receptor at the surface of the target cell. Recent studies have shown, that IL-1 and its signaling pathways play an important role in tumorigenesis and cancer progression [22]. IL-1 $\beta$ can induce EMT process in cells by two ways. In one of these pathways, IL- $1 \beta$ combines with IL-1R 1 and $\beta$-Catenin 
to form the IL-1 $\beta / \mathrm{IL}-1 \mathrm{R} 1 /$ Beta-Catenin; on the other hand, IL- $1 \beta$ increases the expression of Twist 1 transcription factor by activating the PI3K/AKT signaling pathway; which plays an important role in the EMT process. It can subsequently induce the phenomenon of chemo resistance.

Also, Twist 1 transcription factor produced in the downstream of the above pathway can lead to estrogen receptor methylation (ER1). By causing this epigenetic change, it reduces ERa receptor expression and causes resistance to Tamoxifam. The ER1 is the target of Tamoxifam drug [23].

On the other hand, IL- 1 activates the NF-K $\beta$ transcription factor in its downstream signaling. Studies have shown, that NF-K $\beta$ levels are inversely related to the PTEN molecule level in a person with malignancy and chemo resistance; it means, that NF-K $\beta$ is high and PTEN is low in these people. IL-1 increases miR-21 transcription by activating the NF-K $\beta$ transcription factor in people with malignancy. This microRNA inhibits cellular apoptosis by suppressing the expression of PTEN molecule.

Subsequently, Caspase 3 can lead to chemo resistance induction in cited individuals [24]. In COVID-19 patients, IL-1 increases following cytokine storm. Considering the role of IL-1 to induce chemotherapy resistance in cancer patients with COVID-19, it is hypothesized the survival rate reduces and the treatment process is challenged.

\section{Tumor necrosis factor-alpha (TNF- $\alpha$ )}

TNF can be involved in activating, inducing function and differentiating the immune regulatory cells, such as Myeloid-derived Suppressor cell and the Regulatory T cell through the use of TNF-R2. It also plays an impressive role in cancer cells differentiation and tumorigenesis induction.

It also activates the Snail transcription factor through the AKT/GSK3 $\beta /$ Snail signaling pathway, which is involved in EMT process; subsequently, the chemo resistance phenomenon is induced in tumor cells [25].TNF can also induce immune check point molecules expression, such as PDL1 and CD73 in cancer cells. The expression of these molecules is also effective to induce chemo resistance [26]. Thus, NF-K $\beta$ transcription factor increases PDL1 expression, and consequently increases PDL1 expression on the surface of tumor cells by binding to its receptor; PD1 on the surface of Cytotoxic T lymphocyte (CTL) reduces the inhibitory function of CTL on tumor cells.

Through the ERK and mTOR signaling pathway, it can induce proliferation, tumor cells survival increment, and resistance to chemotherapy drugs. So, blocking the PDL1 signaling pathway using monoclonal antibodies is effective to improve treatment process with chemotherapy drugs in people with malignancy [27].
Downstream of the MAPK signaling pathway, the C-jun/ AP-1 transcription factor also increases the CD73 molecule expression. CD73 can also increase the number of tumor cells through HGF/Met/ERK1 pathway. Subsequently, increased expression of CD73 through A2B Adenosine Receptor, and increased expression of Multi Drug Resistance Associated Protein 1 (MDR P1) molecule can lead to chemo resistance [28].

Some studies have shown, that despite the tumorigenesis role of TNF molecule, it can also induce apoptosis by binding to TNFR1 on the surface of tumor endothelial cells; it also plays an important role in the process of chemo sensitivity. By increasing the expression of TNF-related apoptosis inducing ligand (TRAIL) and TRAIL receptors, such as DR4 and DR5 on the surface of cancer cells, chemotherapy drugs increase Caspase8; subsequently, it induces apoptosis. Therefore, it can play a positive role in the process of treatment with chemotherapy drugs [29-31].

TNF can also act as an antitumor molecule, by activating the CTLs and tumor-infiltrating macrophages and dendritic cells (DCs), and thereby lead to chemo sensitivity [32]. It is elevated in COVID-19 infected individuals following a cytokine storm. However, due to its dual role in inducing chemotherapy resistance and creating a positive effect on chemotherapy, more studies are needed to decide about its effect on the process of chemotherapy in cited patients.

\section{Aberrant function of innate immune cells}

\section{Monocyte and macrophage}

Monocytes are components of mononuclear phagocytic system, that play an important role in regulating immune responses. These cells can also be effective to induce cytokine storm by releasing pro-inflammatory cytokines; they can induce cytokine storm in COVID-19 patients, too [5]. Monocytes are also impressive in generating chemo resistance in cancer patients.

Surrounding tumor cells are a number of cells, including Tumor Associated Macrophages (TAMs) and BoneMarrow derived macrophages (BM-DMs). TAMs play an important role in tumor cells growth and inducing resistance to chemotherapy drugs. BM-DMs infiltrate tumor cells through DAMP, CCL2, and CSF-1 molecules; they are also called M1-like macrophages. Tumor cells secrete cytokines, such as IL4, IL10, IL13, and lactic acid in association with $\mathrm{CD} 4+(\mathrm{Th} 2) \mathrm{T}$ cells, leading to M1 to M2 macrophage polarization $[33,34]$.

M2 macrophages can also play an important role in proliferation, angiogenesis, and EMT induction in tumor cells by secreting factors such as EGF, VEGF, and PDGF; it subsequently leads to their resistance to chemotherapy 
drugs [35, 36]. Secreted EGF by M2 macrophages induces RAS/RAF/MAPK, PI3K/AKT and PLC/PKC/CAMK signaling in the downstream of EGF/EGFR signal transmission pathway in the tumor cells, and via binding to the EGFR receptor on the surface of cited cells; it subsequently can be effective to create survival and proliferation of malignant cells through the mentioned signaling pathways [37] (Fig. 1).

In addition, EGF can induce EMT in the tumor cells via ERK1/2-phospho-Smad2/3-Snail signaling pathway [38, 39] (Fig. 1). VEGF can also lead to angiogenesis in TME by binding to its receptor called VEGFR; it acts via the PI3K/ AKT signaling pathway. Subsequently by TGF- $\beta /$ Smad and TGF- $\beta /$ Snail/Slug signaling pathways, EMT is induced in tumor cells; EMT process plays an effective role in the resistance of tumor cells to chemotherapy drugs [40, 41].

On the other hand, PDGF secreted by M2 macrophages can also lead to angiogenesis by utilizing the TGF- $\beta$ signaling pathway. It also induces EMT in tumor cells by Notch1/ Twist 1 pathway, and finally induces chemotherapy resistance [40, 42]. In general, EGF, VEGF and PDGF can induce EMT, and chemotherapy resistance in cancer cells through the TGF- $\beta /$ Smad pathway. Therefore, this common pathway can be used to create appropriate therapies to reduce the effect of chemotherapy resistance [43-45]. Considering the role of monocyte to induce cytokine storm in COVID19 patients, and also its role to induce chemo resistance in cancer patients, it seems they can worsen patient's condition.

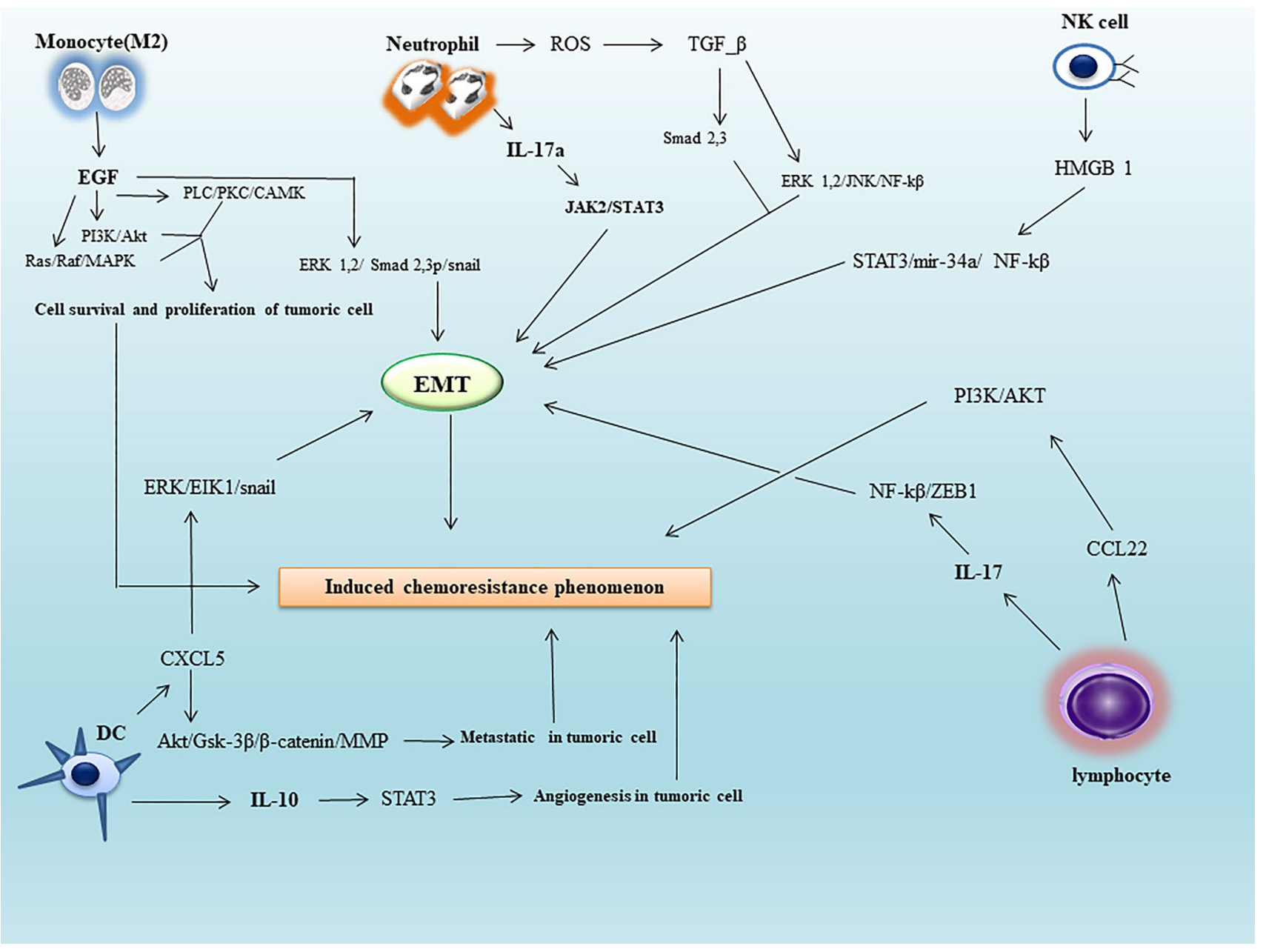

Fig. 1 The role of cytokine-induced immune cells in resistance to chemotherapy drugs in cancer cells. Cancer cells via EMT, cell survival, angiogenesis, proliferation, and cytokine storm-induced metastatic power can inhibit chemotherapy drugs. It leads to the chemo resistance phenomenon in people with cancer. Therefore, timely detection of signaling pathways during the cytokine storm can minimize the occurrence of chemo resistance in people with COVID-19. Abbreviation: $T M E$ tumor microenvironment, $E G F$ epidermal growth factor, $P K C$ protein kinase C, $P I 3 K$ phosphatidylinositol-4,5-bisphosphate 3-kinase, PLC phospholipase C, MAPK mitogen-activated protein kinase, ERK extracellular signal-regulated kinase, $R O S$ reactive oxygen species, $T G F-\beta$ transforming growth factor-beta, $J A K$ just another kinase, STAT signal transducer and activator of transcription, $N F-K \beta$ nuclear factor kappa light chain enhancer of activated B cells, $H M G B 1$ high mobility group box 1, miR microRNA, EMT epithelialmesenchymal transition, $M M P$ matrix metalloproteinase 
Therefore, activation of monocytes can be prevented by providing the appropriate cell therapies; it consequently reduces the induction of cytokine storm and chemotherapy resistance in COVID-19 cancer patients, and will improve the process of treatment.

\section{Neutrophils}

Neutrophils are important cells in defense against microorganisms; they are one of the essential cells in the innate immune responses. In addition, neutrophils in people with COVID-19 may be a part of process to induce cytokine storm. After calling neutrophils to inflamed alveolar cells and exposure to SARS-COV2, they can induce cytokine storm through the formation of Neutrophil Extra Cellular Trap (NET) network [46].

Studies have also shown, that neutrophils are involved in chemotherapy resistance induction in people with cancer. Neutrophils called Tumor Associated Neutrophil (TAN) can secrete IL-17 and release Reactive Nitrogen Species (RNS) and Reactive Oxygen Species (ROS) in TME; they also form a NET network to induce EMT in tumor cells. These processes lead to cancer cells progression and chemotherapy resistance induction [47].

TAN cells secreted IL17a in the TME binds to the IL17R receptor on the surface of tumor cells; IL17a can induce EMT in the tumor cells using JAK2/STAT3 signaling pathway in the downstream of this receptor [48]. Produced ROS by TAN cells lead to the activation of extracellular matrixassociated TGF- $\beta$ latent complex. By activating the latent TGF- $\beta$ complex on tumor cells surface, and then its binding to T $\beta R I$ and T $\beta R I I$ receptors on cell surface, it activates and phosphorylates the Smad 2,3 molecules in the downstream of these receptors, which can induce EMT process in tumor cells (Fig. 1).

Also in another path called non Smad signaling pathway, which causes TGF- $\beta$ binding to its receptors in the downstream, using molecules such as ERK1,2/JNK/NF-K $\beta$ can start EMT process in tumor cells (Fig. 1). On the other hand, the activity of NOXs increases through the NF-K $\beta$ pathway; consequently, this molecule can also produce ROS from tumor cells and leads to a synergistic effect on ROS produced by TANs. Thereby, it increases EMT induction in tumor cells [49].

RNS secreted by TANs can also lead to phosphorylation and activation of the CREB molecule by Protein Kinase A in tumor cells; this molecule can also induce EMT through these pathways [50]. In tumor cells, the CREB molecule activates an IncRNA, called HULC; this IncRNA can induce angiogenesis and EMT by suppressing miR-107 and miR200a-3P. miR-107 suppression increases the efficiency of E2F1/SPHK1 molecules, and finally these molecules lead to angiogenesis in the tumor cells.
Suppression of miR-200a-3P also increases ZEB1 transcription factor activity; in the next step, EMT process occurs in the tumor cells. On the other hand, HULC IncRNA can induce proliferation in the tumor cells in cooperation with P18 molecule [51]. As a result, by inducing the processes of angiogenesis, EMT and tumor cells proliferation increment, chemo resistance also occurs in these cells. In addition, the formed NET network in the TME medium can activate the EMT process in tumor cells via TGF- $\beta$ signaling pathway [52].

Considering the role of neutrophils to induce chemotherapy resistance and cytokine storm creation, it is hypothesized that, their function can worsen the COVID-19 cancer patient's condition. In other words, neutrophil count increment in these patients is a sign of low survival; it also complexes the treatment process.

\section{Dendritic cells}

Dendritic cell (DC) is a professional antigen presenting cell (APC), that plays a key role in relationship between innate and acquired immune responses. DCs can also be effective in inflammation induction. Of course, their role in inflammation is effective on T-helper cells polarization. In COVID-19 patients, DCs are also indirectly involved in cytokine storm induction; they affect $\mathrm{T}$ helper cells [53].

Studies have shown, that DCs play an important role to induce chemo resistance in patients with malignancy. In tumor medium, DCs called Tumor-Associated Dendritic Cell (TADCs) are present, which are effective in angiogenesis, metastasis and EMT induction in tumor cells; in this way, they can cause resistance to chemotherapy drugs [54]. They are also impressive in the progression of cancer cells by secreting CCL5 chemokine, and inducing MALT1/ Snail signaling pathway in tumor cells [55]. DCs can secrete IL-10; it can also induce angiogenesis in TME, by inducing IL10/STAT signaling pathway [56] (Fig. 1).

In addition, these cells can secrete CXCL5 chemokine in TME medium. By CXCL5binding to CXCR2 receptor on the surface of cancer cells, CXCL5 can induce two important signaling pathways; they are effective to induce EMT and metastasis in tumor cells. Thus, in ERK/Elk1/Snail pathway, the EMT process occurs in cancer cells, and in another pathway called AKT/GSK3 $\beta / \beta$-Catenin/MMP, metastasis occurs in cancer cells [57] (Fig. 1).

CXCL1e is another chemokine secreted by DCs in TME; it plays an important role in the metastasis and EMT induction in tumor cells [54]. It induces the NF-K $\beta / S O X 4$ and ERK/MMP2,9 signaling pathways by binding to its receptor on the surface of tumor cells; mentioned pathways play vital roles in tumor cell metastasis, migration and invasion [58, 59]. Also, by connecting CXCL chemokine to CXCR4, AKT 
signaling pathway is activated in the downstream of which is the fork head box class (FOX) molecule.

The FOX molecule can activate the Wnt signaling pathway due to T-cell Factor (TCF)/Beta-Catenin/FOX complex; it leads to EMT induction in cancer cells [60]. In TME and under acidic conditions, some cancer cells secrete TGF- $\beta 2$ molecules; they bind to the TGF $\beta$ R 1 , leading to TGF- $\beta$ / Smad 2,3 pathway induction. Downstream of this pathway, the enzyme diacylglycerol O-acyltransferase (DGAT) is activated, which leads to the accumulation of lipid particles in the DCs called Lipid Droplet (LD); it prevents DCs activation and migration to the lymph nodes to induce TCD8 ${ }^{+}$ function, and remove present tumor cells in TME. It inhibits tumor cells suppression by immune system [61].

Therefore, by suppressing the DCs in TME, they perform their role as the antigen presenters to produce TCD8+ cells. TCD8+ can remove tumor cells and secretes chemokines and other soluble substances, which give the ability to induce EMT and metastatic power to tumor cells. DCs induce resistance to chemotherapy in TME due to TCD8+effects.

Considering the cited roles of DCs, they will worsen the condition of cancer patients. Therefore, using appropriate cell therapies a useful treatment strategy can be presented in COVID-19 patients suffering malignancy, to overcome the phenomenon of chemotherapy resistance.

\section{NK cells (natural killer cell)}

NK cells are in the primary line of defense against viral pathogens. They can also induce cytokine storm in COVID19 infected patients. NK cells also play a vital role to induce resistance to chemotherapy in cancer patients. They lead to tumor cells progression through the High Mobility Group Box1 (HMGB1) molecule, which in turn induces chemo resistance [62, 63]. HMGB1 secretion induces STAT3/ miR-34a/NF-K $\beta$ signaling pathway in tumor cells; subsequently this pathway leads to angiogenesis and EMT process in tumor cells [64] (Fig. 1). In another pathway induced by HMGB 1 in tumor cells, it can play a decisive role in tumor cell metastasis by interacting with the Brahma-Related Gene 1 (BRG1) molecule, and activating AKT signaling pathway $[65,66]$.

NK cells secrete TGF- $\beta$ and macrophage migration inhibitory factor (MIF) in the tumor environment; they are also effective to induce EMT in tumor cells. TGF- $\beta$ induces Snail 1,2, Id1 and Runx 2 transcription factors activity, via the Smad 2,3/Smad 4 molecule in the downstream of own signaling pathway; they are also important to set up EMT process in tumor cells. Finally, TGF-beta can induce EMT in tumor cells [67]. TGF- $\beta$ can also induce migration and invasion in the tumor cells by activating the EGFR receptor through Smad3/ERK/SP1 signaling pathway; cited processes lead to chemotherapy resistance through tumor cells aggression $[68,69]$.

The secreted MIF molecule can induce Akt signaling pathway in tumor cells by binding to the CXCR4. It induces EMT in the tumor cells, using EMT associated transcription factors (EMT-TF). Also, EMT-TF can induce angiogenesis in TME by decreasing E-cadherin expression, and increasing $\mathrm{N}$-cadherin and Vimentin expression. Angiogenesis and EMT play an effective role to induce resistance to chemotherapy drugs [70]. According to the cited facts, it seems NK cells will progress disease and increase mortality in patients.

\section{Aberrant function of adaptive immune cells}

Lymphocytes are the main executive cells in adaptive immune responses. They are involved in cytokine storm induction in COVID-19 patients, and can also induce resistance to chemotherapy drugs in tumor cells. In the tumor medium, T lymphocytes secrete cytokines such as IL-17 and IL-13; the second one causes resistance to chemotherapy drugs [71]. These cytokines induce Akt signaling pathway in tumor cells. This signaling pathway using Snail transcription factor can induce EMT process in tumor cells; it also leads to VEGF production through IL-6 upregulation and subsequent application of JAK2/STAT3 signaling pathway in the downstream of IL-6 (Akt dependent IL-6/JAK2/STAT3). VEGF plays an important role in inducing angiogenesis in TME [72].

IL-17 is also impressive in migration and invasion of tumor cells through NF-K $\beta / Z E B 1$ signaling pathway; it also induces EMT process [73] (Fig. 1). Therefore, by inducing the EMT process and angiogenesis in TME medium, chemotherapy resistance occurs. IL-13 can also induce EMT and chemotherapy resistance via STAT6 signaling pathway [74].

CXCL13 is a T lymphocytes secreted chemokines in TME; it is also effective to induce resistance to chemotherapy drugs [75]. It can induce two MEK/ERK and Rac-GEF signaling pathways by binding to the CXCR5 on the surface of tumor cells, which result in resistance to chemotherapy drugs. MEK/ERK also leads to proliferation of tumor cells, and Rac-GEF signaling pathway is effective in cancer cells migration.

In addition to the two signaling pathways, CXCL13 binding of T lymphocytes to CXCR5 on the surface of tumor cells via PI3K/Akt signaling pathway can be effective to increase the survival of tumor cells; it is also important in cancer progression and increasing drug resistance [76].

B lymphocytes in TME medium are also effective in chemotherapy resistance creation. Thus, secretion of CCL22 chemokine from B lymphocytes can lead to tumor cells resistance to chemotherapy, through CCL22/PI3K/ Akt signaling pathway [77] (Fig. 1). B lymphocytes are also 
important in the phenomenon of drug resistance through CXCR4/CXCL12 signaling pathway [78]. By CXCL12 binding to CXCR4, JAK2/STAT3 signaling pathway is generated in their downstream, which results in STAT3 phosphorylation by the JAK2 enzyme.

Phosphorylated STAT3 molecule can lead to BCL-XL, BCL2 and Cyclin D1 molecules transcription in tumor cells; they inhibit apoptosis and induce proliferation by cell cycle advancing; the product of which is the development of resistance to chemotherapy drugs [79]. It is hypothesized, that lymphocytes may worsen the clinical condition in COVID19 cancer patients.

In addition to immune cells, other cells may be also important in inducing cytokine storms in patients with COVID-19; one of the most important of these cells is fibroblasts [80]. They can be effective in promoting cancer and resistance to chemotherapy by inducing cytokine storm in the cited population. IL- 8 and the CXCL8 chemokine secreted by active fibroblasts bind to CXCR2 on the surface of cancer cells. In the next step, Prostaglandin J2 molecule can activate Snail, Slug, and ZEB1 transcription factors in the downstream of CXCR2 signaling pathway.

Cited transcription factors by binding to the E-box of the E-cadherin molecule, lead to downregulation of this molecule; they can induce EMT in cancer cells. Since EMT can play an important role in inducing the chemo resistance phenomenon in cancer cells, the consequence of the above signaling pathway is chemo resistance development in cancer patients with COVID-19. Also, the ZEB1 transcription factor, which is activated in downstream of this signaling pathway, can inhibit apoptosis induction of chemotherapy drugs by binding to the P53 molecule. Finally, P53 degradation leads to drug resistance in cancer patients with COVID-19 [81, 82].

\section{Conclusion}

Considering the role of immune cells and pro-inflammatory cytokines in the occurrence of cytokine storm and subsequent induction of resistance to chemotherapy (Tables 1 and 2), it is important to identify the signaling pathways. The cytokine storm is effective in the development of severe form of the disease in cancer patients infected with SARS-COV2 virus; by identifying these pathways and providing appropriate treatment strategies the incidence of disease can be reduced in cancer people with COVID-19.

It is important to note, when the patient is healthy after the end of cytokine storm, it will not have any effects on cancer treatment process. Therefore, only at the beginning of the cytokine storm and before its end, signaling pathways induction can have a negative effect on the treatment response in cancer patients, and also affect survival rate of these patients. So, early detection of cytokine storm in cancer patients with COVID-19 is also important to maximize the effectiveness of target therapy.

Table1 Summary of some cytokines involved in the chemotherapy resistance

\begin{tabular}{|c|c|c|c|c|}
\hline Cytokine & Type of disease & Mechanism & Potential drug & Refs. \\
\hline IL-6 & ovarian cancer & IL-6 induced chemo resistance is dependent on the PYK2 phosphorylation & Carboplatin & [83] \\
\hline IL-6 & NSCLC & $\begin{array}{l}\text { IL-6 in the STAT3 pathway leads to the TIMP molecule expression, and thus can } \\
\text { induce drug resistance }\end{array}$ & Gemcitabine & {$[84]$} \\
\hline IL-1 & Pancreatic Cancer & $\begin{array}{l}\text { Tumor-Stroma IL-1 } \beta \text {-IRAK4/NF-K } \beta \text { pathway induces chemo resistance in the } \\
\text { PDAC patient }\end{array}$ & Paclitaxel & {$[85,86]$} \\
\hline IL-1 & AML & $\begin{array}{l}\text { IL1 } \beta / \text { Cox-2/PGE2/ } \beta \text {-Catenin/ARC pathway induces drug resistance in acute } \\
\text { myeloid leukemia (AML) }\end{array}$ & Cytarabine & [87] \\
\hline TNF-a & Breast cancer & $\begin{array}{l}\text { TNF- } \alpha \text { /NTF-ERK-GST- } \pi \text { axis and TNF- } \alpha \text { /NTF-NF- } \kappa \text { B- mediated anti-apoptotic } \\
\text { functions are required for TNF- } a \text {-induced DOX-resistance }\end{array}$ & 6-Mercaptopurine & [88] \\
\hline TNF-a & Breast cancer & $\begin{array}{l}\text { The MEK5/Erk5 pathway through the SNAIL2 and ZEB1 molecules expression can } \\
\text { induce EMT, and consequently drug resistance }\end{array}$ & Docetaxel & {$[89,90]$} \\
\hline TGF- $\beta$ & Colorectal cancer & $\begin{array}{l}\text { Lack of SMAD } 4 \text { molecule in the downstream of TGF- } \beta \text { signaling can induce drug } \\
\text { resistance by activating PI3K/Akt/CDC2/Survivin pathway }\end{array}$ & Vincristine & {$[91,92]$} \\
\hline TGF- $\beta$ & NSCLC & $\begin{array}{l}\text { Simultaneous over activation of Wnt } / \beta \text {-catenin and TGF } \beta \text { signaling by miR-128-3p } \\
\text { confers chemo resistance }\end{array}$ & Cisplatin & [93] \\
\hline
\end{tabular}

IL-6 interleukin-6, NSCLC non-small cell lung carcinoma, TIMP-1 tissue inhibitor of metalloproteinase-1, STAT3 signal transducer and activator of transcription 3,IL-1 interlukin-1, IRAK4 interleukin-1 receptor associated kinase $4, N F-K \beta$ nuclear factor-k $\beta, P D A C$ pancreatic ductal adenocarcinoma, $A M L$ acute myeloid leukemia, $A R C$ apoptosis repressor with caspase recruitment domain, $C O X-2$ cyclooxygenase-2, $P G E 2$ prostaglandin 2, TNF- $\alpha$ tumor necrosis factor alpha, NTF N-terminal fragment, ERK extracellular signal-regulated kinase, GST- $\pi$ glutathione S-transferase- $\pi, D O X$ doxorubicin, $M E K$ mitogen-activated protein kinase kinase, ZEB1 $\delta$-EF1, TGF- $\beta$ transforming growth factor beta, $P I 3 K$ phosphoinositide 3-kinase, Akt serine threonine kinase, $C D C 2$ cell-division cycle 2, WNT wingless-related integration site, SMAD4 SMAD family member 4, EMT epithelial-mesenchymal transition, $m i R$ MicroRNA 
Table 2 Some of chemotherapy drugs involved in cytokines expression

\begin{tabular}{|c|c|c|c|}
\hline Chemotherapy & Cytokine & Mechanism & Refs. \\
\hline Daunorubicin & IL-3 & $\begin{array}{l}\text { IL-3 can induce ERK pathway. ERK/Mcl-1 pathway can inhibit BCL2 protein activity, and } \\
\text { lead to drug resistance }\end{array}$ & [94] \\
\hline Cyclophosphamide & IL-6 & IL6 can induce drug resistance via JAK/STAT3 and NF-kappaB IL6-dependent pathway & [95] \\
\hline Taxotere & IL-8 & $\begin{array}{l}\text { IL-8 can induce drug resistance and cancer progression, through PLC-PKC, PI3K-AKT, Rho- } \\
\text { GTPase family, FAK/Src and MAPK cascade signals and NF- } \mathrm{kB} \text { pathway }\end{array}$ & [96] \\
\hline Methyl methane sulfonate & TGF- $\beta$ & $\begin{array}{l}\text { TGF- } \beta \text { can induce drug resistance through miR } 21 \text { expression and subsequent suppression of } \\
\text { MSH } 2 \text { mRNA production }\end{array}$ & [97] \\
\hline 5-Fluorouracil & $\mathrm{OSM}^{*}$ & OSMRs/JAK1/STAT3 axis contributes to the resistance to the targeted drugs in cancer cells & {$[95,98]$} \\
\hline Methotrexate & IL-17 & $\begin{array}{l}\text { IL-17 leads to the production of KEAP1-Nrf2 and NF-KB transcription factors, through PI3K/ } \\
\text { AKT/mTOR pathway; it can induce drug resistance by expressing the BCRP molecule }\end{array}$ & {$[99,100]$} \\
\hline Etoposide & IL-11 & IL-11 induces resistance to chemotherapy drugs, through gp130/JAK/STAT3/Bcl2 pathway & [101] \\
\hline Oxaliplatin & CXCL1 & Drug resistance is induced via $\mathrm{CXC}-\mathrm{Chemokine} / \mathrm{NF}-\kappa \mathrm{B}$ signaling pathway & [102] \\
\hline
\end{tabular}

Abbreviation: $I L$ interleukin, ERK extracellular signal-regulated kinase, $M c l-1$ myeloid cell leukemia 1, Bax Bcl-2-associated X protein, JAK Janus kinase, STAT signal transducer and activator of transcription $3, N F-K \beta$ nuclear factor-k $\beta, P L C$ phospholipase $\mathrm{C}, P K C$ protein kinase C, $P I 3 K$ phosphoinositide 3-kinase, Akt serine threonine kinase, $F A K$ focal adhesion kinase, $S r c$ short for sarcoma, MAPK mitogen-activated protein kinase, $T G F-\beta$ transforming growth factor beta, $m i R$ MicroRNA, MSH2 MutS homolog 2, OSM oncostatin-M (* IL-6 family cytokine), OSMR OSM receptor, $m T O R$ mammalian target of rapamycin, BCRP breast cancer resistance protein, KEAP1 Kelch-like ECH-associated protein 1, Nrf2 nuclear factor erythroid 2-related factor 2, gp glycoprotein, $B C L-2 \mathrm{~B}$-cell lymphoma 2, $C X C L \mathrm{C}$-X-C motif chemokine ligand

Acknowledgements We wish to thank all our colleagues in Iranian Blood Transfusion Organization.

Author contributions M.A has conceived the manuscript and revised it. M.H, M.M, M.S, M.B, S.A wrote the manuscript.

Data availability This is a review study, and it is not an original. Data availability is corresponding author responsibility.

\section{Declarations}

Conflict of interest The authors declare that they have no conflict of interest.

Ethical approval This article does not contain any studies with human participants or animals performed by any of the authors.

\section{References}

1. Carfî A, Bernabei R, Landi F (2020) Persistent symptoms in patients after acute COVID-19. JAMA 324(6):603-605

2. Hamidian Jahromi A, Mazloom S, Ballard D (2020) What the European and American health care systems can learn from China COVID-19 epidemic; action planning using purpose designed medical telecommunication, courier services, homebased quarantine, and COVID-19 walk-in centers. Immunopathol Persa 6(2):e17. https://doi.org/10.34172/ipp.2020.17. By failing to prepare, you are preparing to fail. 2020.

3. Mehta P, McAuley DF, Brown M, Sanchez E, Tattersall RS, Manson JJ (2020) COVID-19: consider cytokine storm syndromes and immunosuppression. The Lancet 395(10229):1033-1034

4. Sinha P, Matthay MA, Calfee CS (2020) Is a "cytokine storm" relevant to COVID-19? JAMA Intern Med 180(9):1152-1154

5. Guo C, Li B, Ma H, Wang X, Cai P, Yu Q et al (2020) Single-cell analysis of two severe COVID-19 patients reveals a monocyte-associated and tocilizumab-responding cytokine storm. Nat Commun 11(1):1-11

6. Daminov B, Abdullaev S, Sharapov O, Igamberdieva R (2021) Epidemiological, clinical and morphological aspects of kidney damage in COVID-19. J Renal Injury Prev 10(3):e17

7. Henderson LA, Canna SW, Schulert GS, Volpi S, Lee PY, Kernan KF et al (2020) On the alert for cytokine storm: immunopathology in COVID-19. Arthritis Rheumatol 72(7):1059-1063

8. Xia Y, Jin R, Zhao J, Li W, Shen H (2020) Risk of COVID-19 for patients with cancer. Lancet Oncol 21(4):e180

9. Ragab D, Salah Eldin H, Taeimah M, Khattab R, Salem R (2020) The COVID-19 cytokine storm; what we know so far. Front Immunol 11:1446

10. Roohaninasab M, Sadeghzadeh-Bazargan A, Goodarzi A (2021) Effects of laser therapy on periorbital hyperpigmentation: a systematic review on current studies. Lasers Med Sci. https://doi.org/10.1007/s10103-020-03241-6

11. Coomes EA, Haghbayan H (2020) Interleukin-6 in COVID19: a systematic review and meta-analysis. Rev Med Virol 30(6):1-9

12. Khademi M, Roohaninasab M, Goodarzi A, Seirafianpour F, Dodangeh M, Khademi A (2021) The healing effects of facial BOTOX injection on symptoms of depression alongside its effects on beauty preservation. J Cosmet Dermatol 20(5):1411-1415

13. Bromberg J, Wang TC (2009) Inflammation and cancer: IL-6 and STAT3 complete the link. Cancer Cell 15(2):79-80

14. Jin W (2020) Role of JAK/STAT3 signaling in the regulation of metastasis, the transition of cancer stem cells, and chemoresistance of cancer by epithelial-mesenchymal transition. Cells 9(1):217

15. Preca B-T, Bajdak K, Mock K, Lehmann W, Sundararajan V, Bronsert $P$ et al (2017) A novel ZEB1/HAS2 positive feedback loop promotes EMT in breast cancer. Oncotarget 8(7):11530

16. Velden JLVD, Alcorn JF, Guala AS, Badura EC, Janssen-Heininger YM (2011) c-Jun N-terminal kinase 1 promotes transforming growth factor- $\beta 1$-induced epithelial-to-mesenchymal transition via control of linker phosphorylation and transcriptional activity of Smad3. Am J Respir Cell Mol Biol 44(4):571-581 
17. Seddigh-Shamsi M, Mahali SN, Mozdourian M, Allahyari A, Saeedian N, Emadzade M et al (2020) Investigation of hematological parameters related to the severity of COVID-19 disease in Mashhad, Iran. Immunopathol Persa 7(2):e19-e

18. Weyemi U, Redon CE, Sethi TK, Burrell AS, Jailwala P, Kasoji $M$ et al (2016) Twist1 and Slug mediate H2AX-regulated epithelial-mesenchymal transition in breast cells. Cell Cycle 15(18):2398-2404

19. Lin X, Zhang H, Dai J, Zhang W, Zhang J, Xue G et al (2018) TFF3 contributes to epithelial-mesenchymal transition (EMT) in papillary thyroid carcinoma cells via the MAPK/ERK signaling pathway. J Cancer 9(23):4430

20. Chaidos A, Katsarou A, Mustafa C, Milojkovic D, Karadimitris A (2020) Interleukin 6-blockade treatment for severe COVID19 in two patients with multiple myeloma. Br J Haematol 190(1):e9-e11

21. Sadeghzadeh-Bazargan A, Ghassemi M, Goodarzi A, Roohaninasab M, Najar Nobari N, Behrangi E (2021) Systematic review of low-dose isotretinoin for treatment of acne vulgaris: focus on indication, dosage, regimen, efficacy, safety, satisfaction, and follow up, based on clinical studies. Dermatol Ther 34(1): 14438

22. Apte RN, Dotan S, Elkabets M, White MR, Reich E, Carmi Y et al (2006) The involvement of IL-1 in tumorigenesis, tumor invasiveness, metastasis and tumor-host interactions. Cancer Metastasis Rev 25(3):387-408

23. Jiménez-Garduño AM, Mendoza-Rodríguez MG, UrrutiaCabrera D, Domínguez-Robles MC, Pérez-Yépez EA, AyalaSumuano JT et al (2017) IL-1 $\beta$ induced methylation of the estrogen receptor ERa gene correlates with EMT and chemoresistance in breast cancer cells. Biochem Biophys Res Commun 490(3):780-785

24. Zhang K, Chen J, Chen D, Huang J, Feng B, Han S et al (2014) Aurora-A promotes chemoresistance in hepatocelluar carcinoma by targeting NF-kappaB/microRNA-21/PTEN signaling pathway. Oncotarget 5(24): 12916

25. Zhang Z, Xu J, Liu B, Chen F, Li J, Liu Y et al (2019) Ponicidin inhibits pro-inflammatory cytokine TNF-a-induced epithelialmesenchymal transition and metastasis of colorectal cancer cells via suppressing the AKT/GSK-3 $\beta /$ Snail pathway. Inflammopharmacology 27(3):627-638

26. Reinhardt J, Landsberg J, Schmid-Burgk JL, Ramis BB, Bald T, Glodde $\mathrm{N}$ et al (2017) MAPK signaling and inflammation link melanoma phenotype switching to induction of CD73 during immunotherapy. Can Res 77(17):4697-4709

27. Black M, Barsoum IB, Truesdell P, Cotechini T, MacdonaldGoodfellow SK, Petroff M et al (2016) Activation of the PD-1/ PD-L1 immune checkpoint confers tumor cell chemoresistance associated with increased metastasis. Oncotarget 7(9):10557

28. Peng H, Xue R, Ju Z, Qiu J, Wang J, Yan W et al (2020) Cancerassociated fibroblasts enhance the chemoresistance of CD73+ hepatocellular carcinoma cancer cells via HGF-Met-ERK1/2 pathway. Ann Transl Med 8(14):856

29. Wang X, Lin Y (2008) Tumor necrosis factor and cancer, buddies or foes? 1. Acta Pharmacol Sin 29(11):1275-1288

30. Hasanpour Dehkordi A, Keikhaei B, Bahadoram M, Razmjoo S, Barahman M (2020) Keep the corners; impact of chemotherapy on renal function. J Nephropathol 9(1)

31. Darakhshandeh A, Momenzadeh M (2021) Cachexia and anorexia in cancer; a systematic review. Immunopathol Persa. https:// doi.org/10.34172/ipp.2021.20

32. Wohlleber D, Kashkar H, Gärtner K, Frings MK, Odenthal M, Hegenbarth S et al (2012) TNF-induced target cell killing by CTL activated through cross-presentation. Cell Rep 2(3):478-487

33. Ireland LV, Mielgo A (2018) Macrophages and fibroblasts, key players in cancer chemoresistance. Front Cell Dev Biol 6:131
34. Nokhostin F, Dargahi MalAmir M, Tutunchi S, Rezaeeyan H (2020) Evaluation of prognostic/diagnostic value of Hematological markers in the detection of inflammation in coronavirus disease: a review study. J Adv Med Biomed Res 28(128):171-174

35. Singh S, Chakrabarti R (2019) Consequences of EMT-driven changes in the immune microenvironment of breast cancer and therapeutic response of cancer cells. J Clin Med 8(5):642

36. Pouladzadeh M, Safdarian M, Choghakabodi PM, Amini F, Sokooti A (2021) Validation of red cell distribution width as a COVID-19 severity screening tool. Future Sci OA. https://doi. org/10.2144/fsoa-2020-0199

37. Choudhary KS, Rohatgi N, Halldorsson S, Briem E, Gudjonsson T, Gudmundsson S et al (2016) EGFR signal-network reconstruction demonstrates metabolic crosstalk in EMT. PLoS Comput Biol 12(6):e1004924

38. Kim J, Kong J, Chang H, Kim H, Kim A (2016) EGF induces epithelial-mesenchymal transition through phospho-Smad2/3-Snail signaling pathway in breast cancer cells. Oncotarget 7(51):85021

39. Pouladzadeh M, Safdarian M, Eshghi P, Abolghasemi H, Sheibani B, Choghakabodi PM et al (2021) A randomized clinical trial evaluating the immunomodulatory effect of convalescent plasma on COVID-19-related cytokine storm. Intern Emerg Med. https://doi.org/10.1007/s11739-021-02734-8

40. Nantajit D, Lin D, Li JJ (2015) The network of epithelial-mesenchymal transition: potential new targets for tumor resistance. J Cancer Res Clin Oncol 141(10):1697-1713

41. Zadeh FJ, Akbari T, Samimi A, Davari N, Rezaeeyan H (2020) The role of molecular mechanism of Ten-Eleven Translocation2 (TET2) family proteins in pathogenesis of cardiovascular diseases (CVDs). Mol Biol Rep 47(7):5503-5509

42. Chen J, Yuan W, Wu L, Tang Q, Xia Q, Ji J et al (2017) PDGF$\mathrm{D}$ promotes cell growth, aggressiveness, angiogenesis and EMT transformation of colorectal cancer by activation of Notch1/ Twist1 pathway. Oncotarget 8(6):9961

43. Grande M, Franzen A, Karlsson J-O, Ericson LE, Heldin N-E, Nilsson M (2002) Transforming growth factor- $\beta$ and epidermal growth factor synergistically stimulate epithelial to mesenchymal transition (EMT) through a MEK-dependent mechanism in primary cultured pig thyrocytes. J Cell Sci 115(22):4227-4236

44. Janatmakan F, Nesioonpour S, Zadeh FJ, Teimouri A, Vaziri M (2019) Comparing the effect of clonidine and dexmedetomidine on intraoperative bleeding in spine surgery. Anesthesiol Pain Med 9(1):e83967

45. Rajaei E, Shahbazian N, Rezaeeyan H, Mohammadi AK, Hesam S, Zayeri ZD (2019) The effect of lupus disease on the pregnant women and embryos: a retrospective study from 2010 to 2014 . Clin Rheumatol 38(11):3211-3215

46. Zuo Y, Zuo M, Yalavarthi S, Gockman K, Madison JA, Shi H et al (2021) Neutrophil extracellular traps and thrombosis in COVID-19. J Thromb Thrombolysis 51(2):446-453

47. Wu L, Saxena S, Singh RK (2020) Neutrophils in the tumor microenvironment. Tumor Microenviron. https://doi.org/10. 1007/978-3-030-35723-8_1

48. Wang J, Chen X, Shen D, Ge D, Chen J, Pei J et al (2019) A long noncoding RNA NR_045363 controls cardiomyocyte proliferation and cardiac repair. J Mol Cell Cardiol 127:105-114

49. Murillo MM, Carmona-Cuenca I, Del Castillo G, Ortiz C, Roncero C, Sánchez A et al (2007) Activation of NADPH oxidase by transforming growth factor- $\beta$ in hepatocytes mediates up-regulation of epidermal growth factor receptor ligands through a nuclear factor- $\mathrm{\kappa B}$-dependent mechanism. Biochem $\mathrm{J}$ 405(2):251-259

50. Barlow CA, Kitiphongspattana K, Siddiqui N, Roe MW, Mossman BT, Lounsbury KM (2008) Protein kinase A-mediated CREB phosphorylation is an oxidant-induced survival pathway in alveolar type II cells. Apoptosis 13(5):681-692 
51. Ma Z, Huang H, Xu Y, He X, Wang J, Hui B et al (2017) Current advances of long non-coding RNA highly upregulated in liver cancer in human tumors. Onco Targets Ther 10:4711

52. Bozzini S, Pandolfi L, Vertui V, Frangipane V, Violatto M, De Luigi A et al (2021) Induction of epithelial-mesenchymal transition (EMT) by neutrophil extracellular traps (NETs) as possible molecular mechanism in CLAD. J Heart Lung Transpl 40(4):S151

53. Meftahi GH, Jangravi Z, Sahraei H, Bahari Z (2020) The possible pathophysiology mechanism of cytokine storm in elderly adults with COVID-19 infection: the contribution of "inflameaging." Inflamm Res 69(9):825-839

54. Hsu Y-L, Chen Y-J, Chang W-A, Jian S-F, Fan H-L, Wang J-Y et al (2018) Interaction between tumor-associated dendritic cells and colon cancer cells contributes to tumor progression via CXCL1. Int J Mol Sci 19(8):2427

55. Kan JY, Wu DC, Yu FJ, Wu CY, Ho YW, Chiu YJ et al (2015) Chemokine (C-C motif) ligand 5 is involved in tumor-associated dendritic cell-mediated colon cancer progression through non-coding RNA MALAT-1. J Cell Physiol 230(8):1883-1894

56. Nakamura R, Sene A, Santeford A, Gdoura A, Kubota S, Zapata N et al (2015) IL10-driven STAT3 signalling in senescent macrophages promotes pathological eye angiogenesis. Nat Commun 6(1): 1-14

57. Zhao J, Ou B, Han D, Wang P, Zong Y, Zhu C et al (2017) Tumor-derived CXCL5 promotes human colorectal cancer metastasis through activation of the ERK/Elk-1/Snail and AKT/GSK3 $\beta / \beta$-catenin pathways. Mol Cancer 16(1):1-15

58. Wang N, Liu W, Zheng Y, Wang S, Yang B, Li M et al (2018) CXCL1 derived from tumor-associated macrophages promotes breast cancer metastasis via activating NF- $\mathrm{KB} / \mathrm{SOX} 4$ signaling. Cell Death Dis 9(9):1-18

59. Yang C, Yu H, Chen R, Tao K, Jian L, Peng M et al (2019) CXCL1 stimulates migration and invasion in ER-negative breast cancer cells via activation of the ERK/MMP2/9 signaling axis. Int J Oncol 55(3):684-696

60. Zheng X, Lin J, Wu H, Mo Z, Lian Y, Wang P et al (2019) Forkhead box (FOX) G1 promotes hepatocellular carcinoma epithelial-Mesenchymal transition by activating Wnt signal through forming T-cell factor-4/Beta-catenin/FOXG1 complex. J Exp Clin Cancer Res 38(1):1-15

61. Trempolec N, Degavre C, Doix B, Brusa D, Corbet C, Feron O (2020) Acidosis-induced TGF- $\beta 2$ production promotes lipid droplet formation in dendritic cells and alters their potential to support anti-mesothelioma $\mathrm{T}$ cell response. Cancers 12(5): 1284

62. Kang R, Zhang Q, Zeh HJ, Lotze MT, Tang D (2013) HMGB1 in cancer: good, bad, or both? Clin Cancer Res 19(15):4046-4057

63. Hajsadeghi S, Pazoki M, Pakbaz M, Zeraatian S, Zaeim MA (2020) Aortic valve cusp aneurysm as a result of blood culturenegative infective endocarditis, interesting echocardiographic and surgical images. Echocardiography 37(3):469-471

64. Zhang Y, Ren H, Li J, Xue R, Liu H, Zhu Z et al (2021) Elevated HMGB 1 expression induced by hepatitis $B$ virus $X$ protein promotes epithelial-mesenchymal transition and angiogenesis through STAT3/miR-34a/NF- $\mathrm{kB}$ in primary liver cancer. Am J Cancer Res 11(2):479

65. Lv DJ, Song XL, Huang B, Yu Y-Z, Shu FP, Wang C et al (2019) HMGB1 promotes prostate cancer development and metastasis by interacting with Brahma-related gene 1 and activating the Akt signaling pathway. Theranostics 9(18):5166

66. Hajsadeghi S, Amirfarhangi A, Pakbaz M, Pazoki M, Tanha K (2020) Postinfarction intramyocardial dissection, an interesting case report and systematic review. Echocardiography 37(1):124-131
67. Sancisi V, Gandolfi G, Ragazzi M, Nicoli D, Tamagnini I, Piana S et al (2013) Cadherin 6 is a new RUNX2 target in TGF- $\beta$ signalling pathway. PLoS ONE 8(9):e75489

68. Zhao Y, Ma J, Fan Y, Wang Z, Tian R, Ji W et al (2018) TGF- $\beta$ transactivates EGFR and facilitates breast cancer migration and invasion through canonical Smad3 and ERK/Sp1 signaling pathways. Mol Oncol 12(3):305-321

69. Hajsadeghi S, Pazoki M, Meimand SE, Pouresmaeeli M, Zeraatian S (2021) More is better, not always true: misdiagnosis tamponade in postoperative patient due to thick hemostatic tissue. Echocardiography 38(6):1074-1076

70. Guo X, Xu S, Gao X, Wang J, Xue H, Chen Z et al (2017) Macrophage migration inhibitory factor promotes vasculogenic mimicry formation induced by hypoxia via CXCR4/AKT/EMT pathway in human glioblastoma cells. Oncotarget 8(46):80358

71. Salazar Y, Zheng X, Brunn D, Raifer H, Picard F, Zhang Y et al (2020) Microenvironmental Th9 and Th17 lymphocytes induce metastatic spreading in lung cancer. J Clin Investig 130(7):3560-3575

72. Gu F-M, Li Q-L, Gao Q, Jiang J-H, Zhu K, Huang X-Y et al (2011) IL-17 induces AKT-dependent IL-6/JAK2/STAT3 activation and tumor progression in hepatocellular carcinoma. Mol Cancer 10(1):1-13

73. Gu K, Li M-M, Shen J, Liu F, Cao J-Y, Jin S et al (2015) Interleukin-17-induced EMT promotes lung cancer cell migration and invasion via NF- $\mathrm{kB} / \mathrm{ZEB} 1$ signal pathway. Am J Cancer Res 5(3):1169

74. Cao H, Zhang J, Liu H, Wan L, Zhang H, Huang Q et al (2016) IL-13/STAT6 signaling plays a critical role in the epithelialmesenchymal transition of colorectal cancer cells. Oncotarget 7(38):61183

75. Kobayashi S, Murata K, Shibuya H, Morita M, Ishikawa M, Furu M et al (2013) A distinct human CD4+ T cell subset that secretes CXCL13 in rheumatoid synovium. Arthritis Rheum 65(12):3063-3072

76. Kazanietz MG, Durando M, Cooke M (2019) CXCL13 and its receptor CXCR5 in cancer: inflammation, immune response, and beyond. Front Endocrinol 10:471

77. Wei C, Yang C, Wang S, Shi D, Zhang C, Lin X et al (2019) M2 macrophages confer resistance to 5-fluorouracil in colorectal cancer through the activation of CCL22/PI3K/AKT signaling. Onco Targets Ther 12:3051

78. Shen M, Wang J, Ren X (2018) New insights into tumor-infiltrating B lymphocytes in breast cancer: clinical impacts and regulatory mechanisms. Front Immunol 9:470

79. Wang M, Lin T, Wang Y, Gao S, Yang Z, Hong X et al (2017) CXCL12 suppresses cisplatin-induced apoptosis through activation of JAK2/STAT3 signaling in human non-small-cell lung cancer cells. Onco Targets Ther 10:3215

80. Rowaiye AB, Okpalefe OA, Adejoke OO, Ogidigo JO, Oladipo OH, Ogu AC et al (2021) Attenuating the effects of novel COVID-19 (SARS-CoV-2) infection-induced cytokine storm and the implications. J Inflamm Res 14:1487

81. Choi J, Suh J-Y, Kim D-H, Na H-K, Surh Y-J (2020) 15-Deoxy- $\Delta 12,14$-prostaglandin J2 induces epithelial-to-mesenchymal transition in human breast cancer cells and promotes fibroblast activation. J Cancer Prev 25(3): 152

82. Jiang J, Wang K, Chen Y, Chen H, Nice EC, Huang C (2017) Redox regulation in tumor cell epithelial-mesenchymal transition: Molecular basis and therapeutic strategy. Signal Transduct Target Ther 2(1):1-12

83. Pasquier J, Gosset M, Geyl C, Hoarau-Véchot J, Chevrot A, Pocard $\mathrm{M}$ et al (2018) CCL2/CCL5 secreted by the stroma induce IL-6/PYK2 dependent chemoresistance in ovarian cancer. Mol Cancer 17(1):1-14 
84. Xiao W, Wang L, Howard J, Kolhe R, Rojiani AM, Rojiani MV (2019) TIMP-1-mediated chemoresistance via induction of IL-6 in NSCLC. Cancers 11(8): 1184

85. Zhang D, Li L, Jiang H, Li Q, Wang-Gillam A, Yu J et al (2018) Tumor-stroma IL1 $\beta$-IRAK4 feedforward circuitry drives tumor fibrosis, chemoresistance, and poor prognosis in pancreatic cancer. Can Res 78(7):1700-1712

86. Voloshin T, Alishekevitz D, Kaneti L, Miller V, Isakov E, Kaplanov I et al (2015) Blocking IL1 $\beta$ pathway following paclitaxel chemotherapy slightly inhibits primary tumor growth but promotes spontaneous metastasis. Mol Cancer Ther 14(6):1385-1394

87. Carter BZ, Mak PY, Wang X, Tao W, Ruvolo V, Mak D et al (2019) An ARC-regulated IL1 $\beta / C o x-2 / P G E 2 / \beta-C a t e n i n / A R C$ circuit controls leukemia-microenvironment interactions and confers drug resistance in AML. Can Res 79(6):1165-1177

88. Huang H-Y, Chang H-F, Tsai M-J, Chen J-S, Wang M-J (2016) 6-Mercaptopurine attenuates tumor necrosis factor-a production in microglia through Nur77-mediated transrepression and PI3K/ $\mathrm{Akt} / \mathrm{mTOR}$ signaling-mediated translational regulation. J Neuroinflammation 13(1):1-20

89. Zhou C, Nitschke AM, Xiong W, Zhang Q, Tang Y, Bloch M et al (2008) Proteomic analysis of tumor necrosis factor-a resistant human breast cancer cells reveals a MEK5/Erk5-mediated epithelial-mesenchymal transition phenotype. Breast Cancer Res 10(6):1-14

90. Edwardson DW, Boudreau J, Mapletoft J, Lanner C, Kovala AT, Parissenti AM (2017) Inflammatory cytokine production in tumor cells upon chemotherapy drug exposure or upon selection for drug resistance. PLoS ONE 12(9):e0183662

91. Itatani Y, Kawada K, Sakai Y (2019) Transforming growth factor- $\beta$ signaling pathway in colorectal cancer and its tumor microenvironment. Int J Mol Sci 20(23):5822

92. Zhou L, Tang J, Hu F, Liao Y, Li R, Zhou Y et al (2020) Effects of different levels of TGF- $\beta$ expression and tumor cell necrosis rates in osteosarcoma on the chemotherapy resistance of osteosarcoma. J Bone Oncol 23:100299

93. Cai J, Fang L, Huang Y, Li R, Xu X, Hu Z et al (2017) Simultaneous overactivation of $\mathrm{Wnt} / \beta$-catenin and TGF $\beta$ signalling by miR-128-3p confers chemoresistance-associated metastasis in NSCLC. Nat Commun 8(1):1-19
94. McCubrey JA, Steelman LS, Franklin RA, Abrams SL, Chappell WH, Wong EW et al (2007) Targeting the RAF/MEK/ERK, $\mathrm{PI} 3 \mathrm{~K} / \mathrm{AKT}$ and 553 pathways in hematopoietic drug resistance. Adv Enzyme Regul 47:64

95. Chen W, Qin Y, Liu S (2018) Cytokines, breast cancer stem cells (BCSCs) and chemoresistance. Clin Transl Med 7(1):1-13

96. Li JS, Zhao P, Yang LP, Li Y, Tian YG, Li SY et al (2017) Integrating 3-omics data analyze rat lung tissue of COPD states and medical intervention by delineation of molecular and pathway alterations. Biosci Rep 37:BSR20170042

97. Li Y, Rong G, Kang H (2017) Taxotere-induced elevated expression of IL8 in carcinoma-associated fibroblasts of breast invasive ductal cancer. Oncol Lett 13(3):1856-1860

98. Shien K, Papadimitrakopoulou VA, Ruder D, Behrens C, Shen L, Kalhor N et al (2017) JAK1/STAT3 activation through a proinflammatory cytokine pathway leads to resistance to molecularly targeted therapy in non-small cell lung cancer. Mol Cancer Ther 16(10):2234-2245

99. Dong C, Wu J, Chen Y, Nie J, Chen C (2021) Activation of PI3K/ $\mathrm{AKT} / \mathrm{mTOR}$ pathway causes drug resistance in breast cancer. Front Pharmacol 12:143

100. Lainetti PDF, Leis-Filho AF, Laufer-Amorim R, Battazza A, Fonseca-Alves CE (2020) Mechanisms of resistance to chemotherapy in breast cancer and possible targets in drug delivery systems. Pharmaceutics 12(12):1193

101. Ma J, Song X, Xu X, Mou Y (2019) Cancer-associated fibroblasts promote the chemo-resistance in gastric cancer through secreting IL-11 targeting JAK/STAT3/Bcl2 pathway. Cancer Res Treatm 51(1):194

102. de Porras VR, Bystrup S, Martínez-Cardús A, Pluvinet R, Sumoy L, Howells L et al (2016) Curcumin mediates oxaliplatin-acquired resistance reversion in colorectal cancer cell lines through modulation of CXC-Chemokine/NF- $\mathrm{kB}$ signalling pathway. Sci Rep 6(1):1-17

Publisher's Note Springer Nature remains neutral with regard to jurisdictional claims in published maps and institutional affiliations. 